\title{
A MARTINGALE INEQUALITY AND THE LAW OF LARGE NUMBERS ${ }^{1}$
}

\author{
y. S. CHOW $^{2}$
}

In a recent paper [4], Hájek and Rényi have generalized an inequality of Kolmogorov to the following: If $x_{1}, x_{2}, \cdots, x_{m}$ are independent random variables with

$$
E\left(x_{k}\right)=0
$$

and

$$
E\left(\stackrel{2}{x}_{k}\right)<\infty, \quad(k=1,2, \cdots, m)
$$

and $c_{1} \geqq c_{2} \geqq \cdots>0$, for any $\epsilon>0$

$$
P\left\{\max _{m \geq k \geq 1} c_{k}\left|x_{1}+\cdots+x_{k}\right| \geqq \epsilon\right\} \leqq \frac{1}{\epsilon^{2}} \sum_{1}^{m} c_{k}^{2} E\left(x_{k}^{2}\right) .
$$

The original Kolmogorov's inequality [6] has been extended to a martingale inequality by Lévy [8] and Ville [12] and later to a semimartingale inequality by Doob [3]. In this note we will extend (1) to a semi-martingale inequality which contains Doob's inequality as a special case. As Kolmogorov's inequality is the key to the proof of the law of large numbers for a sequence of independent random variables, we will use our inequality to prove a "law of large numbers" for a martingale, which will be shown to include the extensions of Kolmogorov's law of large numbers for independent random variables [7] made by Brunk [1], Chung [2], Kawata and Udagawa [5], and Prohorov [11], and for dependent random variables made by Lévy [8] and Loève [9].

In the following $(W, F, P)$ will be a probability space, $c_{1}, c_{2}, \ldots$ a nonincreasing sequence of positive numbers, $x_{1}, x_{2}, \cdots$ a sequence of random variables, $y_{k}=x_{1}+x_{2}+\cdots+x_{k}$ and $F_{k}$ the Borel field generated by $x_{1}, x_{2}, \cdots, x_{k}$ for each $k$, and for a random variable $z$ we put $z^{+}=\max (z, 0)$.

THEOREM 1. Let $\left(y_{k}\right)$ be a semi-martingale relative to $\left(F_{k}\right)[3, p .294]$ and $\epsilon>0$. Then

Received by the editors April 6, 1959.

${ }^{1}$ This work was supported by the National Science Foundation under Grant G-2794.

2 Now with International Business Machines Corp., Yorktown Heights, N. Y. 


$$
\begin{aligned}
\epsilon P\left\{\max _{m \geq k \geq 1} c_{k} y_{k} \geqq \epsilon\right\} \leqq & c_{1} E\left(y_{1}^{+}\right)+\sum_{2}^{m} c_{k} E\left(y_{k}^{+}-y_{k-1}^{+}\right) \\
& -c_{m} \int_{\left\{\max _{m \geq k \geq 1} c_{k} y_{k}<\epsilon\right\}} y_{m}^{+} d P \\
\leqq & c_{1} E\left(y_{1}^{+}\right)+\sum_{2}^{m} c_{k} E\left(y_{k}^{+}-y_{k-1}^{+}\right) \\
= & \sum_{1}^{m-1}\left(c_{k}-c_{k+1}\right) E\left(y_{k}^{+}\right)+c_{m} E\left(y_{m}^{+}\right) .
\end{aligned}
$$

To prove (2), let

$$
A=\left\{\max _{m \geq k \geq 1} c_{k} y_{k} \geqq \epsilon\right\},
$$

$A_{k}=\left\{c_{j} y_{j}<\epsilon\right.$ for $\left.1 \leqq j<k ; c_{k} y_{k} \geqq \epsilon\right\}$, and $z_{k}=y_{k}^{+}$for $1 \leqq k \leqq m$. Then $A=\mathrm{U}_{1}^{m} A_{k}$ and $A_{k} \in F_{k}$ for each $k$. Hence

$$
\begin{aligned}
\epsilon P(A)= & \epsilon \sum_{1}^{m} P\left(A_{k}\right) \leqq \sum_{1}^{m} c_{k} \int_{A_{k}} y_{k} d P=\sum_{1}^{m} c_{k} \int_{A_{k}} z_{k} d P \\
= & c_{1} E\left(z_{1}\right)-c_{1} \int_{W-A_{1}} z_{1} d P+\sum_{2}^{m} c_{k} \int_{A_{k}} z_{k} d P=c_{1} E\left(z_{1}\right) \\
& +c_{2} \int_{W-A_{1}}\left(z_{2}-z_{1}\right) d P-c_{2} \int_{W-\left(A_{1} \cup A_{2}\right)} z_{2} d P+\sum_{3}^{m} c_{k} \int_{A_{k}} z_{k} d P .
\end{aligned}
$$

By the semi-martingale property, $\int_{A_{1} \cup A_{2} \cup \cdots \cup A_{k}}\left(z_{k+1}-z_{k}\right) d P \geqq 0$ for each $k$, then

$$
\begin{aligned}
\epsilon P(A) \leqq & c_{1} E\left(z_{1}\right)+c_{2} E\left(z_{2}-z_{1}\right)-c_{2} \int_{W-\left(A_{1} \cup A_{2}\right)} z_{2} d P+\sum_{3}^{m} c_{k} \int_{A_{k}} z_{k} d P \\
\leqq & c_{1} E\left(z_{1}\right)+c_{2} E\left(z_{2}-z_{1}\right)+c_{3} \int_{W-\left(A_{1} \cup A_{2}\right)}\left(z_{3}-z_{2}\right) d P \\
& -c_{3} \int_{W-\left(A_{1} \cup A_{2} \cup A_{3}\right)} z_{3} d P+\sum_{4}^{m} c_{k} \int_{A_{k}} z_{k} d P \\
\leqq & \cdots c_{1} E\left(z_{1}\right)+\sum_{2}^{m} c_{k} E\left(z_{k}-z_{k-1}\right)-c_{m} \int_{W-A} z_{m} d P \\
\leqq & c_{1} E\left(z_{1}\right)+\sum_{2}^{m} c_{k} E\left(z_{k}-z_{k-1}\right) .
\end{aligned}
$$


Thus the proof is complete.

If $x_{1}, x_{2}, \ldots$ are independent with mean zero and finite variance, then $\left(y_{k}^{2}\right)$ is a semi-martingale relative to $\left(F_{k}\right)\left[3\right.$, p. 294] and $E\left(y_{k}^{2}\right)$ $=E\left(x_{1}^{2}+\cdots+x_{k}^{2}\right)$, and therefore (2) reduces to (1). If $c_{k}=1$ for each $k$, then $c_{k}-c_{k+1}=0$ and (2) reduces to Doob's inequality [3, p. 314].

As an application of Theorem 1 we have:

COROLlaRy. Let $\left(y_{k}\right)$ be a non-negative semi-martingale relative to $\left(F_{k}\right)$ and $\lim c_{k}=0$. If for some $\alpha \geqq 1 E\left(y_{k}^{\alpha}\right)<\infty$ for each $k$ and

$$
\sum_{2}^{\infty} c_{k}^{\alpha} E\left(y_{k}^{\alpha}-y_{k-1}^{\alpha}\right)<\infty,
$$

then

$$
\lim c_{k} y_{k}=0 \text { a.e. }
$$

Since $\left(y_{k}^{\alpha}\right)$ is semi-martingale [3, p. 295], by Theorem 1 for $\epsilon>0$, we have

$$
\begin{aligned}
\epsilon^{\alpha} P\left\{\sup _{k \geq n} c_{k} y_{k} \geqq \epsilon\right\} & =\epsilon^{\alpha} P\left\{\sup _{k \geq n} c_{k}^{\alpha} y_{k}^{\alpha} \geqq \epsilon^{\alpha}\right\} \\
& \leqq c_{n}^{\alpha} E\left(y_{n}^{\alpha}\right)+\sum_{n+1}^{\infty} c_{k}^{\alpha} E\left(y_{k}^{\alpha}-y_{k-1}^{\alpha}\right) .
\end{aligned}
$$

By Kronecker's lemma [9, p. 238] and (3),

$$
\lim \underset{c_{n}^{\alpha}}{ } E\left(y_{n}^{\alpha}\right)=0,
$$

and then

$$
\lim P\left\{\sup _{k \geq n} c_{k} y_{k} \geqq \epsilon\right\}=0 .
$$

Hence (4) holds under the condition (3).

When $\left(y_{k}\right)$ is a martingale and $E\left(y_{k}^{2}\right)<\infty$ for each $k,\left(y_{k}^{2}\right)$ is a semimartingale [3, p. 295] and $E\left(y_{k}^{2}\right)=E\left(x_{1}^{2}\right)+\cdots+E\left(x_{k}^{2}\right)[3$, p. 92]. Therefore, if $x_{k}$ 's are uniformly bounded and $c_{k}=1 / k$ the corollary gives Lévy's result [8, p. 252], and if $\alpha=2$ the corollary reduces to Loève's extension $[9, \mathrm{p} .387]$ of Lévy's result.

In the following, $A$ will denote a constant, not necessarily always the same, depending on $\alpha$ and $\beta$.

TheOREM 2. Let $\left(y_{k}\right)$ be a martingale relative to $\left(F_{k}\right), \lim c_{k}=0$, $\alpha \geqq 1$ and $2 \alpha \geqq \beta>0$. If for $i \geqq i_{0}$ 


$$
E\left(\left|y_{i}\right|^{2 \alpha}\right) \leqq A E\left(\sum_{1}^{i} x_{k}^{2}\right)^{\alpha}
$$

$$
i^{\alpha-1} c_{i}^{2 \alpha-\beta} \leqq A, \quad \sum_{i}^{\infty} c_{k}^{2 \alpha} k^{\alpha-2} \leqq A c_{i}^{\beta}
$$

and

$$
\sum_{1}^{\infty} c_{k}^{\beta} E\left(\left|x_{k}\right|^{2 \alpha}\right)<\infty
$$

then (4) is true.

In the proof we may assume that $i_{0}=1$. By the Hölder's inequality,

$$
E\left(\left|y_{k}\right|^{2 \alpha}\right) \leqq A k^{\alpha-1} \sum_{1}^{k} E\left(\left|x_{i}\right|^{2 \alpha}\right) .
$$

By (8), (6) and Kronecker's lemma,

$$
\begin{aligned}
\lim c_{k}^{2 \alpha} E\left(\left|y_{k}\right|^{2 \alpha}\right) & \leqq A \lim c_{k}^{2 \alpha} k^{\alpha-1} \sum_{1}^{k} E\left(\left|x_{i}\right|^{2 \alpha}\right) \\
& \leqq A \lim c_{k}^{\beta} \sum_{1}^{k} E\left(\left|x_{i}\right|^{2 \alpha}\right)=0 .
\end{aligned}
$$

Again by (8),

$$
\begin{aligned}
\sum_{1}^{\infty}\left(c_{k}^{2 \alpha}-c_{k+1}^{2 \alpha}\right) E\left(\left|y_{k}\right|^{2 \alpha}\right) & \leqq A \sum_{1}^{\infty}\left(c_{k}^{2 \alpha}-c_{k+1}^{2 \alpha}\right) k^{\alpha-1} \sum_{1}^{k} E\left(\left|x_{i}\right|^{2 \alpha}\right) \\
& =A \sum_{1}^{\infty} E\left(\left|x_{i}\right|^{2 \alpha}\right) \sum_{i}^{\infty}\left(c_{k}^{2 \alpha}-c_{k+1}^{2 \alpha}\right) k^{\alpha-1} .
\end{aligned}
$$

Now by (6)

$$
\begin{aligned}
\sum_{i}^{\infty}\left(c_{k}^{2 \alpha}-c_{k+1}^{2 \alpha}\right) k^{\alpha-1} & =c_{i}^{2 \alpha} i^{\alpha-1}+\sum_{i+1}^{\infty} c_{k}^{2 \alpha}\left((k+1)^{\alpha-1}-k^{\alpha-1}\right) \\
& \leqq c_{i}^{2 \alpha} i^{\alpha-1}+A \sum_{i+1}^{\infty} c_{k}^{2 \alpha} k^{\alpha-2} \leqq A c_{i}^{\beta} .
\end{aligned}
$$

Hence

$$
\sum_{1}^{\infty}\left(c_{k}^{2 \alpha}-c_{k+1}^{2 \alpha}\right) E\left(\left|y_{k}\right|^{2 \alpha}\right) \leqq A \sum_{1}^{\infty} c_{i}^{\beta} E\left(\left|x_{i}\right|^{2 \alpha}\right)<\infty,
$$

and (4) is true by the corollary.

If $x_{1}, x_{2}, \cdots$ are independent with mean zero, then (5) is satisfied by an inequality due to Marcinkiewicz and Zygmund [10, Theorem 
13]. If there is a subsequence $c_{n_{k}}$ of $c_{n}$ such that $1<r \leqq c_{n_{k}} / c_{n_{k+1}}$ $\leqq r^{\prime}<\infty$ and $c_{k} \leqq A / k$, then (6) is satisfied with $\beta=\alpha+1$, since for $n_{k_{0}} \leqq i<n_{k_{0}}$ we have

$$
i^{\alpha-1} c_{i}^{2 \alpha-\beta} \leqq A
$$

and

$$
\begin{aligned}
\sum_{k=i}^{\infty} c_{k}^{2 \alpha} k^{\alpha-2} & \leqq \sum_{n_{k_{0}}}^{\infty} c_{k}^{2 \alpha} k^{\alpha-2} \leqq \sum_{j=k_{0}}^{\infty} c_{n_{j}}^{2 \alpha} \sum_{k=n_{j}}^{n_{j+1}-1} k^{\alpha-2} \\
& \leqq A \sum_{k_{0}}^{\infty} c_{n_{j} n_{j+1}}^{2 \alpha-1} \leqq A \sum_{k_{0}}^{\infty} c_{n_{j}}^{\alpha+1} n_{n_{j+1}}^{\alpha-1} n_{j+1}^{\alpha-1} \\
& \leqq A \sum_{k_{0}}^{\infty} c_{n_{j}}^{\alpha+1} \leqq A c_{n_{k_{0}}}^{\alpha+1}\left(1+\frac{1}{r}+\frac{1}{r^{2}}+\cdots\right) \leqq A c_{n_{k_{0}}}^{\alpha+1} \\
& \leqq A c_{n_{k_{0}}+1}^{\alpha+1} \leqq A c_{i}^{\alpha+1} .
\end{aligned}
$$

Therefore Theorem 2 includes the results obtained by Brunk, Chung, Kawata and Udagawa, and Prohorov. It is easy to verify that (6) is satisfied by $c_{k}=k^{-r}$ for $r>0$ and $\beta=2 \alpha-(\alpha-1) / r>0$, and by $c_{k}=k^{-k}$ where $\beta$ is any positive number less than $2 \alpha$. The last case, $c_{k}=k^{-k}$, gives an example that the usual condition $\lim \sup c_{k} / c_{k+1}<\infty$ for $\lim c_{k} y_{k}=0$ a.e. for the independent random variable case is not necessary.

\section{REFERENCES}

1. H. D. Brunk, The Strong law of large numbers, Duke Math. J. vol. 15 (1948) pp. 181-195.

2. K. L. Chung, The strong law of large numbers, Proceedings of the Second Berkeley Symposium on Statistics and Probability, 1951, pp. 341-352.

3. J. L. Doob, Stochastic processes, New York, 1953.

4. J. Hájek and A. Rényi, Generalization of an inequality of Kolmogorov, Acta Math. Acad. Sci. Hungar. vol. 6 (1955) pp. 281-283.

5. T. Kawata and M. Udagawa, On the strong law of large numbers, Ködai Math. Sem. Rep. (1951) pp. 78-80.

6. A. Kolmogorov, Über die Summen durch den Zufall bestimmter unabhängiger Grössen, Math. Ann. vol. 99 (1928) pp. 309-319.

7. - Sur loi forte des grands nombers, C. R. Acad. Sci. Paris vol. 91 (1930) pp. 910-912.

8. P. Lévy, Théorie de l'addition des variables aléatories, Paris, 1937.

9. M. Loève, Probability theory, New York, 1935.

10. J. Marcinkiewicz and A. Zygmund, Sur les fonctions indépendents, Fund. Math. vol. 29 (1937) pp. 60-90.

11. U. V. Prohorov, On the strong law of large numbers (Russian), Izv. Akad. Nauk SSSR Ser. Mat. vol. 14 (1950) pp. 523-536.

12. J. Ville, Etude critique de la notion de collectif, Paris, 1939.

\section{UNIVERSITY OF ILLINOIS}

